CLINICAL STUDY

\title{
Comparison of long-acting testosterone undecanoate formulation versus testosterone enanthate on sexual function and mood in hypogonadal men
}

\author{
F Jockenhövel, T Minnemann ${ }^{1}$, M Schubert $^{1}$, S Freude $^{1}$, D Hübler $^{2}, \mathrm{C} \mathrm{Schumann}^{1}$, A Christoph $^{1}$ and M Ernst ${ }^{2}$ \\ Evangelisches Krankenhaus Herne, Wiescherstraße 24, 44623 Herne, Germany, ${ }^{1}$ Klinik II und Poliklinik für Innere Medizin, Klinikum der Universität \\ zu Köln, Kerpener Street 62, 50937 Köln, Germany and ${ }^{2}$ Jenapharm GmbH E Co., KG, Otto-Schott-Straße 15, O7745 Jena, Germany \\ (Correspondence should be addressed to F Jockenhövel; Email: f.jockenhoevel@evk-herne.de)
}

\begin{abstract}
Objective: To compare the effects of two treatment modalities of testosterone on sexual functioning and mood.

Design: Forty men were randomized to receive either parenteral testosterone enanthate (TE) or longacting parenteral testosterone undecanoate (TU) over a period of 30 weeks. Thereafter, 20 men who had received TU and 16 men who had received TE continued with TU and completed another 65 weeks to study longer-term effects of TU.

Methods: The following variables of sexual functioning were studied: sexual thoughts and fantasy, sexual interest and desire, satisfaction with sex life, number of erections and ejaculations per week, and number of spontaneous morning erections per week. Also variables related to mood were analyzed. Results: Improvements in these variables were significant and were of a similar magnitude in the group treated with TU and TE for 30 weeks. Improvements were maintained at the same levels over a period of another 65 weeks when all men received TU. Effects on mood were recorded for 30 weeks, but were more difficult to establish in the study population. There were significant differences in baseline values between the two groups and scores showed wide S.D.

Conclusions: Both TE and TU were effective in improving sexual functions in hypogonadal men. An advantage of TU over TE is its lower frequency of administration and its better tolerability and safety profile.
\end{abstract}

European Journal of Endocrinology 160 815-819

\section{Introduction}

Aging is the most robust factor predicting erectile difficulties. It is obvious that aging per se is associated with a deterioration of the biological functions mediating erectile function: hormonal, vascular, and neural processes. This is often aggravated by intercurrent disease in old age, such as diabetes mellitus, cardiovascular disease, and use of medical drugs.

Erectile response in mammals is centrally and peripherally regulated by androgens (1). Severe hypogonadism in men usually results in loss of libido and potency which can be restored by androgen administration. The original insights into the mechanisms of action of androgens on sexual function indicated that androgens exert particular effects on libido and that sleep-related erections are androgen sensitive but erections in response to erotic stimuli are relatively androgen-independent (1). There are a number of recent developments that shed new light on testosterone treatment of erectile dysfunction (ED) in aging men. There is growing insight that testosterone has profound effects on tissues of the penis involved in the mechanism of erection and that testosterone deficiency impairs the anatomical and physiological/biochemical substrate of erectile capacity, reversible upon androgen treatment (2-4). Several studies have indicated that the administration of phospho-diesterase inhibitors type 5 (PDE-5inhibitors) is not always sufficient to restore erectile potency in men, and that administration of testosterone improves the therapeutical response to PDE-5-inhibitors considerably $(3,4)$. There is an increasing insight to view ED occurring in elderly as an expression of the ailments of the aging process. Circulating levels of testosterone are closely related to manifestations of etiological factors in ED, such as atherosclerotic disease and diabetes mellitus (5-7). The latter diseases are correlated with lower-than-normal testosterone levels.

The effects of testosterone on sexual interest and activity are obvious. But treatment of hypogonadal men with testosterone leads almost always to unmistakable changes in mood, self-esteem, and vitality.This testifies to the profound effects of testosterone that it exerts on the brain and the mind. Conversely, hypogonadism and 
particularly the profound hypogonadism resulting from androgen deprivation treatment in men with prostate cancer are associated with loss of vitality and mood disorders, if not depression. It is reasonable to assume that a positive mood state has a favorable effect on sexual functioning.

Testosterone formulations for (i.m.) injection and s.c. application as well as for oral and transdermal administration have been approved for androgen therapy (8). To date, injectable testosterone esters are the most commonly used formulations. To increase serum testosterone levels to the physiological range, i.m. injections of testosterone enanthate (TE) every 2-3 weeks are required, which lead to supraphysiological peaks shortly after administration, followed by a sharp fall in levels thereafter. Testosterone levels before the next injection are frequently in the hypogonadal range (9). Unfortunately, marked oscillations in serum testosterone concentration and short inter-injection intervals of this treatment regimen are associated with considerable discomfort for the patients (9). Therefore, development of longer-acting formulations represents a major improvement in testosterone therapy $(8,10)$.

Testosterone undecanoate (TU), an ester with a fatty acid side-chain of medium length in $17 \beta$-position, is a long-acting formulation for i.m. injection and requires significantly less frequent injections than other established parenteral testosterone ester formulations $(8,11,12)$. After the first injection, a second dose is given 6 weeks later and in the vast majority of patients an injection every 12 weeks (with variations between 11-13 weeks) maintains plasma testosterone in the physiological range (8).

This study assessed the long-term efficacy of i.m. TU for treatment of sexual dysfunction associated with hypogonadism in men. The first part of the study consisted of a 30-week comparative testing of i.m. TU versus standard treatment with i.m. TE. A follow-up study investigated the longer-term effects of TU. Patients, who had completed the comparative study, received now TU for an additional 65 weeks.

The pharmacokinetic aspects of the present study have been published by our group (11).

\section{Subjects and methods}

\section{Study design and patients}

The study was designed as an open-label, randomized, prospective clinical trial (11) to compare testosterone treatment with the traditional parenteral testosterone ester, TE, versus the new parenteral long acting TU and was carried out between October 1998 and February 2002. Forty men were included in the study. Their ages ranged between 18 and 65 years and their serum testosterone levels at inclusion in the present study were $<5 \mathrm{nmol} / \mathrm{l}$ (normal range $10-30 \mathrm{nmol} / \mathrm{l}$ ) following discontinuation of prior testosterone treatment for at least eight weeks, and, if testosterone pellets had been used, for 12 months.

Testosterone therapy had not been received by seven patients previously. To ensure that the patients were meeting the inclusion criteria, they underwent two initial screening visits 42 and 21 days prior to randomization. Only if serum testosterone was $<5 \mathrm{nmol} / \mathrm{l}$ on both occasions, patients were eligible for inclusion. If medical history, physical examination, and laboratory analysis at screening revealed evidence of severe physical or mental illness, of alcohol or drug abuse or of any contraindication against testosterone treatment (such as severe lower urinary tract symptoms, suspected malignancy of the prostate, erythrocytosis, heart/liver/kidney failure), patients were excluded from participation in the study. All patients gave their written informed consent for inclusion in the study. The study protocols were approved by the Ethics Committee of the University and the State Medical Board, Cologne, Germany.

The study medication (TU (Nebido) $1000 \mathrm{mg}$ in $4 \mathrm{ml}$ castor oil) and Testosteron-Depot JENAPHARM Injektionslösung (TE $250 \mathrm{mg}$ in $1 \mathrm{ml}$ oily solution) were manufactured by Jenapharm $\mathrm{GmbH} \& \mathrm{Co}$., KG, Jena, Germany. All i.m. injections were administered into the gluteus medius muscle, starting on day 0 . The first four TU injections were given at two intervals of six weeks, the following after an interval of nine weeks. All following injections were given at 12-week intervals (18). TE injections were administered at 3-week intervals. Every three weeks during the comparison study, patients presented for blood sampling and assessment of individual study variables.

The patients were randomly assigned (using the SAS software) for treatment with either TU i.m. $(n=20)$ or TE i.m. $(n=20)$ for 30 weeks. There were no essential differences between the two treatment groups regarding age, body mass index, and baseline serum testosterone levels.

After completing the comparison study, all 20 patients of the TU group and 16 patients of the TE group agreed to participate in a follow-up study, wherein all subjects were receiving long acting TU for an additional 65 weeks. Three out of the four patients of the TE group not included in the follow-up study with TU did not consent to be included in a longer-term study and one patient was excluded because of the study protocol violations. Patients who had received TE treatment earlier received the first two TU administrations with an interval of eight weeks, followed by intervals of 12 weeks. Examinations during the followup study were performed every three months, whereas prostate and andrological status were assessed every nine months.

The testosterone preparations used in this study were provided by Jenapharm GmbH \& Co KG. 
Table 1 Serum testosterone values (mean \pm S.D.) before and during administration of parenteral testosterone enanthate (TE) and testosterone undecanoate (TU).

\begin{tabular}{|c|c|c|c|c|c|c|c|c|c|c|}
\hline Weeks & Screen & 0 & 6 & 12 & 18 & 24 & 30 & 45 & 60 & 90 \\
\hline $\begin{array}{l}\text { TE nmol/l } \\
\text { TU nmol/l }\end{array}$ & $\begin{array}{l}3.1 \pm 1.1 \\
2.9 \pm 1.2\end{array}$ & $\begin{array}{l}3.0 \pm 1.3 \\
3.2 \pm 1.4\end{array}$ & $\begin{array}{l}8.3 \pm 7.3 \\
9.5 \pm 3.9\end{array}$ & $\begin{array}{r}9.3 \pm 9.1 \\
15.1 \pm 4.9\end{array}$ & $\begin{array}{r}9.2 \pm 8.7 \\
17.8 \pm 4.1\end{array}$ & $\begin{array}{r}9.7 \pm 7.9 \\
22.4 \pm 6.0\end{array}$ & $\begin{array}{r}9.6 \pm 8.2 \\
17.0 \pm 4.9\end{array}$ & $19.1 \pm 5.1$ & $18.7 \pm 4.9$ & $17.9 \pm 3.9$ \\
\hline
\end{tabular}

For the assessment of possible psychosexual effects a standardized questionnaire published by Behre co-workers (13) based on standardized questionnaires $(14,15)$ was used to assess general mood and sexual activity, as well as frequency of erections and ejaculations and number of morning waking erections, sexual thoughts and fantasies, sexual interest and desire, satisfaction with sexuality, as well as questions of general well-being during the past seven days. These data were collected for three consecutive days before each clinic visit over the first 30 weeks of the study when the effects of TU were compared with those of TE and also for a period of 65 weeks when all men received TU. Over the first 30 weeks of the study when TU was compared with TE, the patients were asked to rate their state concerning 12 different items (sociability, concentration, agitation, self-confidence, listlessness, dizziness, activation, depression, fatigue, anxiety, good mood, and aggressivity). The ratings were performed by the patients using a $10 \mathrm{~cm}$ scale with two extremes designated 'not at all' and 'very strong'. The subjects marked a point between the two extremes. The ratings related to sexual fantasies, sexual interest, and satisfaction with sex life were recorded in the same way.

The answers were evaluated by measuring the distance between the beginning of the line segment ('not at all' and the mark made by the subject, multiplying this value by 100 and dividing by the length of the whole line segment, a method resembling a Likert-type scale, though points were not fixed.

\section{Study design}

The study was performed as an open, randomized, controlled, 2-arm clinical study.

\section{Intention-to-treat analysis}

All patients who had taken one of the study preparations and for whom data from the treatment phase were available were included in the intention-to-treat analysis.

\section{Statistical analysis}

The statistical analysis was carried out by Jenapharm. For this, the software package SAS for Windows NT, Version 6.12 (Statistical Analysis System, SAS Institute, Cary, NC, USA) was used.

\section{Explorative and descriptive data analysis}

The analysis was performed exploratively and descriptively. All variables investigated within the frame of the clinical study were included. Missing values were evaluated as such and were not replaced by estimates. Confidence intervals (95\%) were calculated for selected parameters. Data were displayed separately by examination time and treatment group. Confidence intervals were determined for the difference between the two treatment groups concerning the individual parameters, in order to compare the two treatments.

Table 2 Parameters of sexual behavior (ratings on sexual thoughts/fantasy, sexual interest/desire, satisfaction with sex life, number of ejaculations and erections, total and spontaneous morning erections) in patients with hypogonadism at baseline and after 30 weeks of treatment with testosterone enanthate (TE) and at baseline, after 30 weeks and 95 weeks of treatment with testosterone undecanoate (TU).

\begin{tabular}{|c|c|c|c|c|c|}
\hline \multirow{2}{*}{$\begin{array}{l}\text { Parameter (distance on a } \\
100 \mathrm{~mm}-\mathrm{VAS} \text { in } \mathrm{mm} \text { ) }\end{array}$} & \multicolumn{2}{|c|}{ TE } & \multicolumn{3}{|c|}{ TU } \\
\hline & Screening & Week 30 & Screening & Week 30 & Week 95 \\
\hline Sexual thoughts/fantasy & $31.6 \pm 26.7$ & $56.0 \pm 20.3^{*}$ & $28.5 \pm 28.1$ & $53.9 \pm 28.5^{\star}$ & $52.7 \pm 26.4$ \\
\hline Sexual interest and desire & $27.1 \pm 24.0$ & $51.4 \pm 22.5^{\star}$ & $20.1 \pm 19.0$ & $54.0 \pm 26.2^{*}$ & $53.9 \pm 25.9$ \\
\hline Satisfaction with sex life & $20.2 \pm 26.1$ & $48.3 \pm 23.7^{\star}$ & $9.4 \pm 16.2$ & $50.6 \pm 29.6^{*}$ & $49.8 \pm 25.7$ \\
\hline Number of ejaculations per week (total) & $0.5 \pm 0.6$ & $2.4 \pm 2.6^{\star}$ & $0.8 \pm 1.2$ & $3.2 \pm 2.2^{*}$ & $3.2 \pm 2.1$ \\
\hline Number of erections per week (total) & $0.6 \pm 0.8$ & $5.3 \pm 3.2^{\star}$ & $2.8 \pm 4.1$ & $4.9 \pm 3.4^{*}$ & $4.7 \pm 3.1$ \\
\hline $\begin{array}{l}\text { Number of spontaneous morning } \\
\text { erections per week }\end{array}$ & $0.5 \pm 1.2$ & $3.3 \pm 2.4^{*}$ & $1.5 \pm 2.6$ & $3.2 \pm 2.3^{*}$ & $3.6 \pm 2.7$ \\
\hline
\end{tabular}

VAS, visual analog scale.

${ }^{\star} P<0.05$ versus screening. Scores at week 95 not different from scores at week 30 . 
Table 3 Parameters of general well-being (VAS ratings on 12 items) in patients with hypogonadism at baseline and after 30 weeks of treatment with testosterone undecanoate (TU) (four doses of $1000 \mathrm{mg}$ ) or testosterone enanthate (TE) (10 doses of $250 \mathrm{mg}$ ).

\begin{tabular}{|c|c|c|c|c|}
\hline \multirow[b]{2}{*}{$\begin{array}{l}\text { Parameter (distance on a } \\
100 \mathrm{~mm}-\mathrm{VAS} \text { in } \mathrm{mm} \text { ) }\end{array}$} & \multicolumn{2}{|c|}{ TE } & \multicolumn{2}{|c|}{ TU } \\
\hline & Screening & Week 30 & Screening & Week 30 \\
\hline Sociability & $42.9 \pm 28.3$ & $56.8 \pm 25.6$ & $57.2 \pm 24.2$ & $61.3 \pm 17.7$ \\
\hline Concentration & $36.8+23.9$ & $57.4+20.0^{*}$ & $51.1+20.7$ & $60.7+15.6^{*}$ \\
\hline Agitation & $24.8 \pm 26.5$ & $38.6 \pm 25.9^{*}$ & $36.2 \pm 21.9$ & $53.4 \pm 17.8^{*}$ \\
\hline Self-confidence & $34.5 \pm 19.3$ & $62.2 \pm 17.8^{*}$ & $54.7 \pm 26.1$ & $63.2 \pm 15.8^{*}$ \\
\hline Listlessness & $53.0 \pm 30.7$ & $27.8 \pm 21.2$ & $43.9 \pm 24.7$ & $31.4 \pm 17.0$ \\
\hline Dizziness & $31.4 \pm 31.7$ & $20.5 \pm 17.0^{*}$ & $31.1 \pm 28.6$ & $29.0 \pm 16.9$ \\
\hline Activation & $34.6 \pm 23.1$ & $60.0 \pm 18.8^{*}$ & $45.2 \pm 23.1$ & $56.3 \pm 17.6^{*}$ \\
\hline Depression & $48.1 \pm 30.6$ & $23.4 \pm 25.6$ & $32.8 \pm 27.6$ & $34.7 \pm 23.9$ \\
\hline Fatique & $54.5+30.5$ & $31.8+26.9$ & $55.3+25.3$ & $35.1+20.0$ \\
\hline Anxiety & $37.3 \pm 32.3$ & $21.0 \pm 19.2$ & $32.4 \pm 28.2$ & $27.8 \pm 19.4$ \\
\hline Good mood & $35.1+21.4$ & $62.1+21.4^{\star}$ & $54.4+24.1$ & $59.9+15.7^{*}$ \\
\hline Aggressivity & $31.4 \pm 29.0$ & $28.1 \pm 16.6$ & $31.2 \pm 23.5$ & $26.3 \pm 18.2$ \\
\hline
\end{tabular}

${ }^{*} P<0.05$ versus screening

\section{Results}

Table 1 presents plasma testosterone values \pm s.D. over the study period. Following administration of TE plasma testosterone levels fluctuate strongly evidenced by the large SDS of measured values. At baseline, scores of sexual motivation and performance scores were similar in both groups.

Intramuscular injections of both TU and TE improved all assessed parameters on general mood and sexual activity. There were no significant differences between the two treatment modalities.

Assessed by a standardized questionnaire, there was a significant increase in waking morning erections as well as in total erections and ejaculations (Table 1) per week without significant differences between the two groups. These good results were maintained throughout the treatment with TU for the next 65 weeks. About $50 \%$ of hypogonadal patients in the study had no erections and ejaculations before testosterone administration. The erectile function improved in about $85 \%$ of patients during the androgen replacement therapy in both treatment groups. Only six patients with concomitant severe diseases were non-responders. The long-term i.m. administration of TU improved the ED in another two patients.

Also sexual thoughts and fantasies, sexual interest and desire, and satisfaction with sex life (Table 2) significantly increased during testosterone replacement and continued to be improved significantly in the follow-up with TU injections given in every 12 weeks.

Among the 12 items of subjective mood assessment, agitation, self-confidence, activation, good mood and concentration (Table 3) showed a significant improvement during the treatment and further significant improvement during follow-up with TU treatment. The other items, i.e. sociability, listlessness, dizziness, depression, fatigue, anxiety, and aggressivity (Table 3), improved too, but not significantly. This tendency was the same during the follow-up with treatment with TU.
Self-assessment scores of the parameters of well-being were characterized by high variability. SD values were especially high in screening data (between 19.3 and 32.3 ); in data obtained during week 30 , SD values were from 15.6 to 26.9 .

\section{Discussion}

This study of the relatively novel parenteral testosterone preparation TU compared, in the first instance, effects on a number of androgen-related parameters of sexual functions and mental functions with those of the 'classical' TE over a period of 30 weeks. The effects of TU and TE were largely similar. Subsequently, monotherapy with TU was given for an additional 65 weeks when gains in sexual functioning achieved with either TU or TE over the first 30 weeks were maintained.

There were some differences in the improvement of mental functions in the first 30 weeks of the study, when the effects of TU were compared with those of TE. But these differences were quantitatively small and mostly based on the poorer initial scores of patients randomly assigned to the TE group. So, scores of the group treated with TE were poorer than the group treated with TU but the groups were dissimilar with regard to baseline scores preventing reliable conclusions as the superiority of TU over TE in this regard or the converse.

This study report does not address safety issues of the two types of parenteral testosterone administration; these data have been published earlier $(16,17)$.

\section{Conclusion}

Administration of TU every 12 weeks is at least as efficacious for treatment of sexual complaints of hypogonadal men as TE. These improvements are maintained in the longer-term. While being at least as 
effective as the standard injectable formulation, treatment with TU requires only four injections per year while maintaining serum testosterone levels within the physiological range. There are data to confirm the safety and efficacy of long-term TU therapy in hypogonadal patients treated over a period of more than eight years (18). TU appears to be a safe modality of testosterone treatment, because with the presently established dosage regimen, plasma testosterone levels remain in the physiological range. With TU, there is almost never an occurrence of polycythemia as observed in studies with the more traditional testosterone esters (19-21).

\section{Declaration of interest}

The authors declare that there is no conflict of interest that could be perceived as prejudicing the impartiality of the research reported.

\section{Funding}

This research did not receive any specific grant from any funding agency in the public, commercial or not-for-profit sector.

\section{References}

1 Bancroft J. The endocrinology of sexual arousal. Journal of Endocrinology 2005186 411-427.

2 Gooren LJ \& Saad F. Recent insights into androgen action on the anatomical and physiological substrate of penile erection. Asian Journal of Andrology 20068 3-9.

3 Traish AM \& Guay AT. Are androgens critical for penile erections in humans? Examining the clinical and preclinical evidence. Journal of Sexual Medicine 20063 382-407 discussion 387-404.

4 Rochira V, Balestrieri A, Madeo B, Granata AR \& Carani C. Sildenafil improves sleep-related erections in hypogonadal men: evidence from a randomized, placebo-controlled, crossover study of a synergic role for both testosterone and sildenafil on penile erections. Journal of Andrology 200627 165-175.

5 Shabsigh R, Arver S, Channer KS, Eardley I, Fabbri A, Gooren L, Heufelder A, Jones H, Meryn S \& Zitzmann M. Sexual health as a portal to men's health: a problem turned around into an opportunity. International Journal of Clinical Practice 200862 179-181.

6 Shabsigh R, Katz M, Yan G \& Makhsida N. Cardiovascular issues in hypogonadism and testosterone therapy. American Journal of Cardiology 200596 67M-72M.

7 Shabsigh R, Arver S, Channer KS, Eardley I, Fabbri A, Gooren L, Heufelder A, Jones H, Meryn S \& Zitzmann M. The triad of erectile dysfunction, hypogonadism and the metabolic syndrome. International Journal of Clinical Practice 200862 791-798.

8 Nieschlag E. Testosterone treatment comes of age: new options for hypogonadal men. Clinical Endocrinology $200665275-281$.

9 Behre HM, Wang C, Handelsman DJ \& Nieschlag E. Pharmacology of testosterone preparations. In Testosterone, Action, Deficiency, Substitution, pp 405-444. Eds E Nieschlag \& HM Behre, UK: Cambridge University Press, 2004.
10 Nieschlag E, Behre HM, Bouchard P, Corrales JJ, Jones TH, Stalla GK, Webb SM \& Wu FC. Testosterone replacement therapy: current trends and future directions. Human Reproduction Update $200410409-419$.

11 Schubert M, Minnemann T, Hubler D, Rouskova D, Christoph A, Oettel M, Ernst M, Mellinger U, Krone $\mathrm{W}$ \& Jockenhovel F. Intramuscular testosterone undecanoate: pharmacokinetic aspects of a novel testosterone formulation during long-term treatment of men with hypogonadism. Journal of Clinical Endocrinology Metabolism 200489 5429-5434.

12 Harle L, Basaria S \& Dobs AS. Nebido: a long-acting injectable testosterone for the treatment of male hypogonadism. Expert Opinion on Pharmacotherapy 20056 1751-1759.

13 Behre HM, Nashan D, Hubert W \& Nieschlag E. Depot gonadotropin-releasing hormone agonist blunts the androgeninduced suppression of spermatogenesis in a clinical trial of male contraception. Journal of Clinical Endocrinology Metabolism 1992 74 84-90.

14 Zerssen von D. Die Beschwerden-Liste Weinheim, Germany: Beltz, 1976.

15 Jahnke W \& Debus G. Die Eigenschaftsworterliste EWL Eine mehrdimensionale Methode zur Beschreibung von Aspekten des Befindens Goettingen, Germany: Hogrefe, 1978.

16 Minnemann T, Schubert M, Hubler D, Gouni-Berthold I, Freude S, Schumann C, Oettell M, Ernst M, Mellinger U, Sommer F, Krone W \& Jockenhovel F. A four-year efficacy and safety study of the longacting parenteral testosterone undecanoate. Aging Male 200710 155-158.

17 Minnemann T, Schubert M, Freude S, Hubler D, Gouni-Berthold I, Schumann C, Christoph A, Oettel M, Ernst M, Mellinger U, Krone $\mathrm{W} \&$ Jockenhovel F. Comparison of a new long-acting testosterone undecanoate formulation versus testosterone enanthate for intramuscular androgen therapy in male hypogonadism. Journal of Endocrinological Investigation $2008 \mathbf{3 1}$ 718-723.

18 Saad F, Kamischke A, Yassin A, Zitzmann M, Schubert M, Jockenhel F, Behre HM, Gooren L \& Nieschlag E. More than eight years' hands-on experience with the novel long-acting parenteral testosterone undecanoate. Asian Journal of Andrology 2007 9 291-297.

19 Jockenhovel F, Vogel E, Reinhardt W \& Reinwein D. Effects of various modes of androgen substitution therapy on erythropoiesis. European Journal of Medical Research 19972 293-298.

20 Dobs AS, Meikle AW, Arver S, Sanders SW, Caramelli KE \& Mazer NA. Pharmacokinetics, efficacy, and safety of a permeationenhanced testosterone transdermal system in comparison with bi-weekly injections of testosterone enanthate for the treatment of hypogonadal men. Journal of Clinical Endocrinology Metabolism 199984 3469-3478.

21 Calof OM, Singh AB, Lee ML, Kenny AM, Urban RJ, Tenover JL \& Bhasin S. Adverse events associated with testosterone replacement in middle-aged and older men: a meta-analysis of randomized, placebo-controlled trials. Journals of Gerontology Series A: Biological Sciences and Medical Sciences 200560 1451-1457.

Received 29 January 2009

Accepted 15 February 2009 